\title{
Yellow-green and amber InGaN micro-pixellated light-emitting diode arrays
}

\author{
Z. Gong, D. Massoubre, E.Y. Xie, J. \\ McKendry, E. Gu, and M.D. Dawson \\ Institute of Photonics \\ University of Strathclyde \\ Glasgow G4 0NW, U.K. \\ Zheng.gong@strath.ac.uk
}

\author{
N.Y. Liu, Y.B. Tao, \\ Z.Z. Chen, and \\ G.Y.Zhang \\ Department of Physics \\ University of Peking \\ Beijng 100871, China
}

\author{
Y.B. Pan, and M.S. Hao \\ Epilight Technology Co., Ltd. \\ Shanghai 201203, \\ China.
}

\begin{abstract}
Micro-pixel InGaN LED arrays operating at $560 \mathrm{~nm}$ and $600 \mathrm{~nm}$, respectively, are demonstrated, based on new epitaxial structures. Such devices have applications in areas including bioinstrumentation, visible light communications and micro-displays.
\end{abstract}

\section{INTRODUCTION}

The development of efficient InGaN light-emitting diodes (LEDs) at visible wavelengths longer than green $(>540 \mathrm{~nm})$ is a topic of great importance, particularly for white light illumination and full color display applications [1] and involves major materials challenges that are the subject of much current research. Yellow-green and amber wavelengths also offer great potential for instrumentation, communications and microdisplay applications, for which the micro-pixellated array form of InGaN LED ('micro-LED') has shown particular relevance [2]. Here we report, to the best of our knowledge, the first demonstration of micro-pixellated InGaN LED arrays made from yellow green $(560 \mathrm{~nm})$ and amber $(600 \mathrm{~nm})$ wafers, the crystal quality of which being suitable for applications-level device performance. The success of these micro-LED devices paves the way towards a variety of promising applications such as full-colour micro-displays [3], optoelectronic tweezers (OET) [4] and optogenetics with optogenetic inhibitors [5]. For example, green wavelengths give optimized carrier generation from a-Si photoconductive electrodes used in OET, offering the prospect of integrated and multi-functional optoelectronic tweezer devices; Halorhodopsin (NpHR) optogenetic inhibitors are optimally excited by yellow-amber light, offering, with our device format, chip-scale devices for optogenetics..

\section{EXPERIMENTS}

The yellow-green $(560 \mathrm{~nm})$ and amber $(600 \mathrm{~nm}) \mathrm{InGaN}$ wafers were grown by MOCVD on (0001) sapphire substrates. Details of the full wafer structures will be reported elsewhere. Demonstrator devices have been made in two formats. A 64X64 matrix-addressable LED array, in which each microdisk pixel has a $20 \mu \mathrm{m}$ emission aperture on a $50 \mu \mathrm{m}$ pitch, has been made by standard semiconductor processing techniques from the yellow-green wafer. For this device, all pixels in the same row share a common p-contact, and all pixels in the same column share a common n-electrode. In this way, each pixel in the 64X64 array can be addressed separately. The detailed fabrication procedure follows that of similar matrix devices with shorter wavelengths [3]. A 10X10 individuallyaddressable micro-LED array, in which each pixel has a $30 \mu \mathrm{m}$ emission aperture on a $100 \mu \mathrm{m}$ pitch, has been made from the amber wafer. For this device, each pixel has an independent pcontact and a corresponding metal track connected to the pixel, whereas all pixels share a common n-contact. Optical images of a portion of the yellow-green 64X64 matrix device and amber 10X10 LED array are shown in Fig.1(a), and Fig.1(b) respectively.

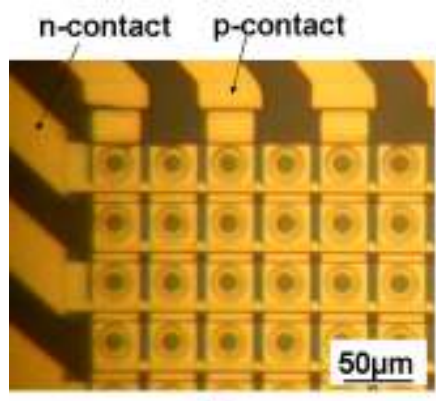

(a)

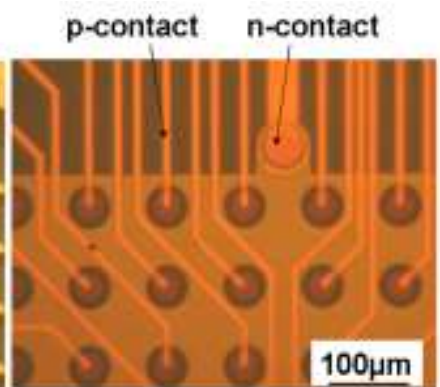

(b)
Fig.1 (a) optical microscope images of part of (a) the yellow-green matrix device, and (b) the 10X10 amber InGaN LED array.

\section{RESULTS}

Fig. 2(a) shows the representative I-V curve of a single pixel in the 64X46 yellow-green matrix array. The turn-on voltage is around $3.6 \mathrm{~V}$, slightly higher than for a conventional broad-area LED fabricated from the same wafer. The inset in Fig. 2(a) shows a typical electroluminescence (EL) spectrum of one pixel under a forward bias of $4.3 \mathrm{~V}$, showing the emission wavelength is $560 \mathrm{~nm}$ with a full spectral width at half maximum of $45 \mathrm{~nm}$. Optical power of $2.5 \mu \mathrm{W}$ per pixel is achieved at an injection current of $2 \mathrm{~mA}$, as shown in Fig. 2(b). This corresponds to a power density of $\sim 0.8 \mathrm{~W} / \mathrm{cm}^{2}$, which however, will be much higher if a larger injection current is applied. Fig.2(c) shows the optical image of one pixel under operation, illustrating that it can be addressed separately. Computer-controlled patterns can be also generated by the matrix yellow green devices, as shown in Fig. 3(d). This is 

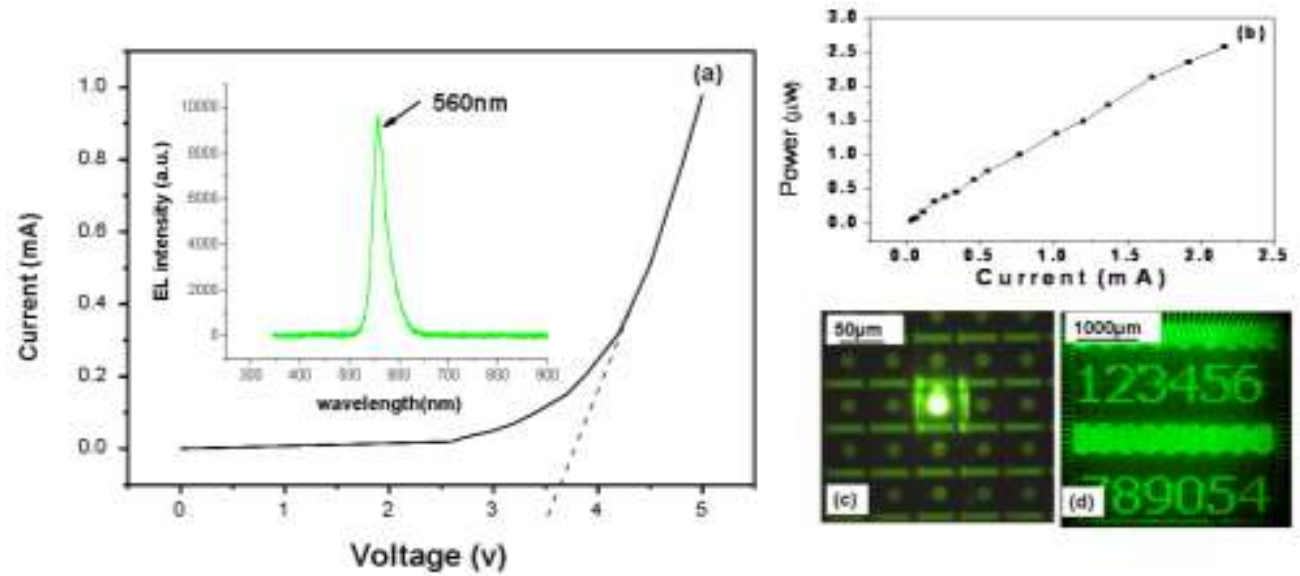

Fig.2. (a) I-V curve of one individual pixel from the yellow-green matrix device. Inset shows the EL spectrum at a bias of 4.3V. (b) power output as a function of the injection current; (c) optical image of one pixel turned on; (d) programmable patterns generated onto the matrix device by using a driver circuit.

achieved by driving the LED array with a suitable driver circuit under a raster scanning mode and a corresponding Labview ${ }^{\mathrm{TM}}$ control interface [3]. Such programmable emission patterns are ideally suited for a wide range of applications, including bioinstrumentation. Similarly, we have characterized the device performance of the amber 10X10 LED array. The typical I-V curve of one individual pixel from the 10X10 LED array is shown Fig. 3(a). The peak emission wavelength is $\sim 600 \mathrm{~nm}$, as shown in the inset in Fig. 3(a). This emission wavelength however, is shifted to shorter wavelength with increasing forward bias (not shown), which we attribute to the strong piezoelectric field induced by the high indium composition in the quantum well. Typical power output from one single pixel is $5.5 \mu \mathrm{W}$ at an injection current of $15 \mathrm{~mA}$, as shown in Fig. 3 (b). The corresponding power density is up to $0.78 \mathrm{~W} / \mathrm{cm}^{2}$, high enough for those applications mentioned earlier. Optical images of one, two, and three pixels in operation are shown in Fig.3(c), illustrating that each pixel can be individuallyaddressable.

\section{CONCLUSIONS}

The successful demonstration of these LED array opens up numerous applications such as optical particles trapping, fullcolor displays, and electrophysiology [2-5].

\section{REFERENCES}

[1] Chen et al., IEEE Photon. Technol. Lett. Vol 18, No.21, 2006, pp.22692271

[2] M.D. Dawson, and M.A.A. Neil, Cluster issue on "Micro-pixellated LED's for science and instrumentation“, J. Phys. D: Appl. Phys. 2008, 41, p.090301.

[3] Gong et al., IEEE Trans. Electron. Devices, Vol.54, No.27, 2007, pp2650-2657

[4] Chiou et al., Nature, Vol.436, 2005, pp.370-372.

[5] Scanziani et al., Nature, Vol.461, 2009, pp.930-939
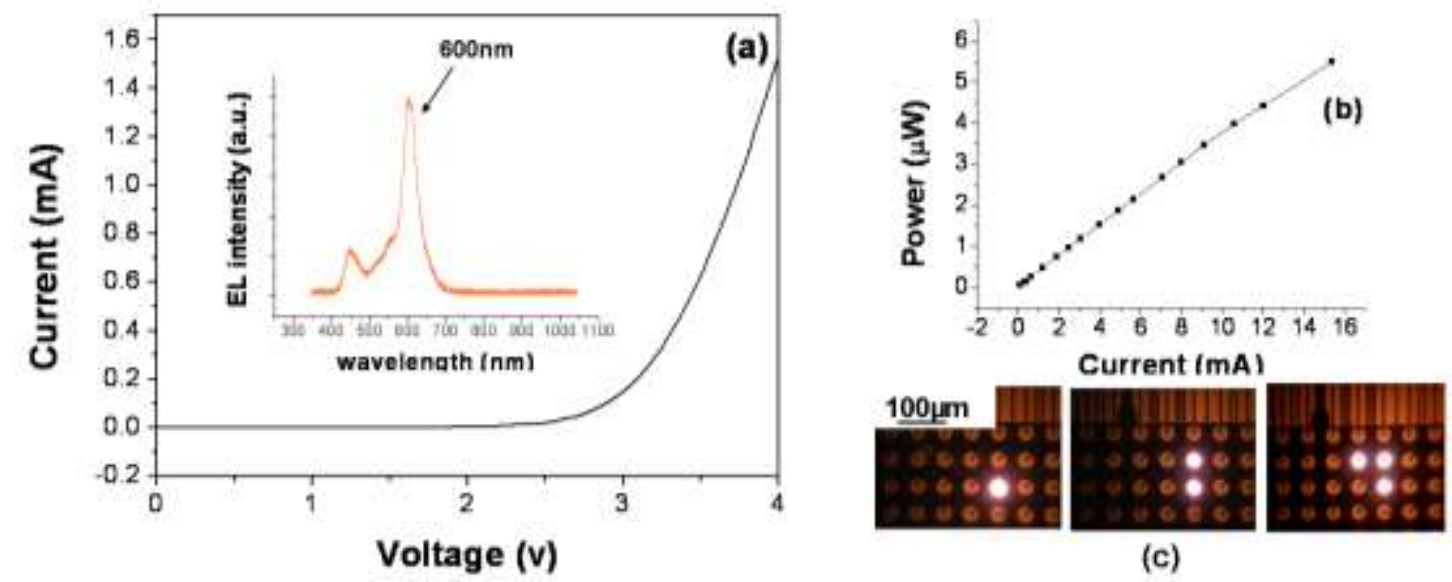

(c)

Fig.3. (a) typical I-V curve of one individual pixel from the amber 10X10 LED array. Inset shows the EL spectrum at a bias of 4V. (b) power output as a function of the injection current; (c) optical image of one pixel, two pixels, and three pixels turned on 